\title{
Time and Temperature Dependent Damage Characteristics of 5083 Al Alloy under Cavitation-Corrosion Condition
}

\author{
S.J. LEE ${ }^{a}$, M.S. HAN ${ }^{b}$, J.Y. JEONG ${ }^{c}$, M.J. KIM ${ }^{a}$ AND S.J. KIM ${ }^{b, *}$ \\ ${ }^{a}$ Kunsan National University, Department of Power System Engineering, Daeha-ro 558 Gunsan-si, Korea \\ ${ }^{b}$ Mokpo National Maritime University, Division of Marine System Engineering, \\ Haeyangdaehak-Ro 91 Mokpo-si, Korea \\ ${ }^{c}$ Mokpo National Maritime University, Division of Navigation Science, Haeyangdaehak-Ro 91 Mokpo-si, Korea

\begin{abstract}
Continuous efforts to construct high-speed and lightweight ship have been made to reduce cost against growing oil prices. In this context, aluminum has received attention as an alternative material for steel, for small and medium-sized ships. In an effort to extend service life of aluminum, various researches have been attempting to improve the corrosion resistance and anti-corrosion characteristics by applying heat treatment and alloying. The material, however, has a high probability of being attacked by corrosion and erosion, caused by high ship speed in severe marine environments. Cavitation damage is dependent on several important factors, such as shape of objects, surface roughness, rotation speed, fluid pressure and velocity. Vapor pressure, density and surface tension of fluid are also taken into account. In this study, an electrochemical cavitation erosion-corrosion experiment was conducted as a function of solution temperature and applied amplitude for 5083-O aluminum alloy used commercially as a hull material for a small sized ship. The result of the experiment revealed that the damage was accelerated due to synergistic effect of corrosion and erosion as the solution temperature was elevated, and that the weight loss showed a steady increase with increasing time of cavitation.
\end{abstract}

DOI: 10.12693/APhysPolA.129.747

PACS/topics: 47.55.dp, 82.45.Bb

\section{Introduction}

The hull of aluminum ship is vulnerable to erosion damage induced by wave impact pressure in the case of sailing with high speed. This damage tends to be aggravated greatly due to the synergistic effect of erosion and corrosion by chloride ions, a severe corrosion factor in sea water. As a way of preventing damage from corrosion and erosion in marine environments, application of anti-corrosion materials, cathodic protection, and corrosion inhibitor are highly recommended, and the related researches are in progress on this subject [1]. These approaches, however, are unable to determine the precise mechanism of damage, since they draw conclusions from the analysis of two individual damage processes [2]. In the relevant research, Hiromi et al. investigated the cavitation erosion of pure titanium and titanium alloys using a rotating ring method. They have revealed that cavitation resistance is increased with the increasing hardness [3]. Chen et al. conducted an experiment of vibration cavitation-erosion using $40 \mathrm{Cr}$ steel to investigate the damage mechanism of cavitation-erosion. They have found complete pits, incomplete pits and thermal pits [4]. Kim et al. researched the effect of manganese and boron on cavitation behavior of austenite steel. They have shown that both elements enhance resistance to cavitation [5]. In the research carried by Brujan et al.,

*corresponding author; e-mail: ksj@mmu.ac.kr cavitation-erosion experiment was performed in polymer solution. They have confirmed that minor damage is induced, as compared to that of salt water [6]. This study proposes an integrated approach to determining more precise damage mechanism, considering the synergistic effect of corrosion and cavitation erosion in sea water. A testing apparatus for the electrochemical cavitation erosion-corrosion experiment was devised for this purpose.

\section{Experimental methods}

In this study, 5083-O Al alloy was used as the ecofriendly material due to its good weldability and formability and high corrosion resistance. Table I shows the chemical composition of this alloy. A schematic diagram of the apparatus for potentiostatic tests in cavitation environments is shown in Fig. 1.

TABLE I

Chemical compositions of 5083-O Al alloy.

\begin{tabular}{c|c|c|c|c|c|c|c|c}
\hline \hline $\mathrm{Si}$ & $\mathrm{Fe}$ & $\mathrm{Cu}$ & $\mathrm{Mn}$ & $\mathrm{Mg}$ & $\mathrm{Cr}$ & $\mathrm{Zn}$ & $\mathrm{Ti}$ & $\mathrm{Al}$ \\
\hline 0.19 & 0.30 & 0.06 & 0.74 & 4.06 & 0.11 & 0.05 & 0.01 & $\mathrm{Bal}$.
\end{tabular}

Electrochemical cells were made using potentiostatic/galvanostatic equipment. The cavitation and electrochemical characteristics of the cells were assessed in sea water. The test bath contained working electrodes (5083-O), reference electrodes $(\mathrm{Ag} / \mathrm{AgCl})$, and counterelectrodes (platinum). Cavitation experiment was conducted with opposite vibration method of the vibration generating device based on the piezoelectric effect. 




Fig. 1. Schematic diagram in potentiostatic experiment under cavitation condition.

It generates a rated output of $20 \mathrm{kHz}$ and supplies it to the vibrator. The amplitude of vibrations was set to $10 \mu \mathrm{m}$ by static amplitude automatic control. For generation of vibration by piezoelectric device, electric alternating current was applied to a conical horn to generate vibration in the axial direction. The sample was fixed to the support so that it was facing the horn of the vibrator, and the distance of $1 \mathrm{~mm}$ was maintained using a filler gauge. An electronic balance that can measure down to $10^{-4} \mathrm{~g}$ was used to measure weights before and after the test, to calculate the weight loss and cavitation rate after washing the sample with an ultrasonic washer and drying it in a vacuum dryer for 24 hours. To analyze the surface damage behavior after cavitation test, a scanning electron microscope and a $3 \mathrm{D}$ microscope were used.

\section{Results and discussion}

Figure 2 compares the natural potential of 5083-O aluminum alloy at different sea water temperature, at the amplitude of $10 \mu \mathrm{m}$.

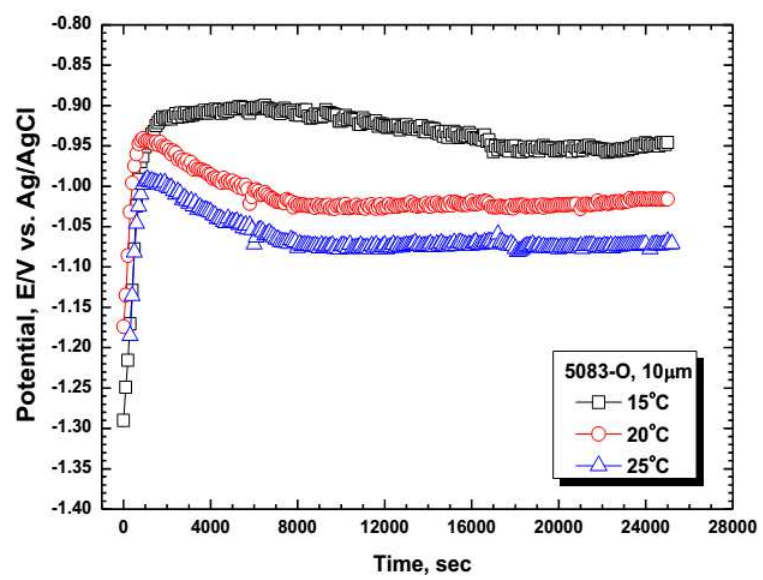

Fig. 2. Comparison of natural potential of 5083-O Al alloy at $10 \mu \mathrm{m}$ amplitude, at different sea water temperature.
Overall, the potential has shown a sudden rise at the early stage of immersion, and then stabilized. Formation of passive film such as $\mathrm{Al}_{2} \mathrm{O}_{3}$ or $\mathrm{Al}(\mathrm{OH})_{3}$ in sea water contributes to this potential change. At the completion of experiment, the highest potential was observed at $15^{\circ} \mathrm{C}$, and the lowest potential at $25^{\circ} \mathrm{C}$. The maximum potential difference was $-0.123 \mathrm{~V}$. The effect of solution temperature on damage cannot be simply determined, because there are several factors being influenced by the temperature including properties of fluid, amount of dissolved gas, and properties of material. It is, however, known that the damage rate is reduced at low temperatures owing to low viscosity and high surface tension [7]. In the case of mild steel, the damage rate in $3 \% \mathrm{NaCl}$ solution is slowed down by either an increase or decrease in temperature from $50^{\circ} \mathrm{C}$. It is so, because the amount of the dissolved oxygen increases at low temperatures, while the vapor pressure increases at high temperatures [7]. In this study, the difference in cavity impact pressure caused by control of temperature is considered very small, because the temperatures were much lower, and the difference was minor. This well corresponds to the result of Park, according to which, the influence of water temperature on cavitation damage is strong, however under $40^{\circ} \mathrm{C}$, the temperature does not affects the damage by impact. In terms of corrosion, however, charge transfer is more active at higher temperatures, and in this case mobilization of $\mathrm{Cl}^{-}$ion is activated, resulting in a potential shift in the active direction.

Figure 3 presents anodic polarization and cathodic polarization curves of 5083-O aluminum alloy at sea water temperature at the vibration amplitude of $10 \mu \mathrm{m}$.

On the anodic polarization curve in Fig. 3a, the open circuit potential was varied from $-1.106 \mathrm{~V}$ to $-0.982 \mathrm{~V}$. At the early stage of polarization, short-term fluctuation of current density appeared due to low impressed potential and disturbance of sea water generated by cavities. For each temperature, there existed a region where the increase in current density had slowed down. The potential was varied from $0.228 \mathrm{~V}$ to $0.374 \mathrm{~V}(-0.982$ to $-0.608 \mathrm{~V}$ at $15^{\circ} \mathrm{C},-1.106$ to $-0.756 \mathrm{~V}$ at $20^{\circ} \mathrm{C}$, and -0.971 to $-0.743 \mathrm{~V}$ at $25{ }^{\circ} \mathrm{C}$ ) with a slight difference, showing a tendency for increase with the increasing temperature. The reason for such behavior is that the corrosion products of oxidation reaction are firmly established on the surface and prevent the charge transfer. In this case, however, the oxidation product on the surface was removed by oxygen evolution due to continuous active dissolution reaction as well as delamination of grain boundary, indicating the increasing tendency in current density. A similar tendency was generally observed, however a higher current density at the same level of potential was observed with the increasing temperature. With the increase of temperature, the passive potential region narrows, and the current density and corrosion rate decrease [8]. In this respect, the lowest temperature of $15^{\circ} \mathrm{C}$ is expected to present the highest resistance to corrosion. On the cathodic polarization curve in Fig. 3b, 



Fig. 3. Comparison of anodic (a) and cathodic (b) polarization curves for 5083-O Al alloy at different sea water temperature, at vibration amplitude of $10 \mu \mathrm{m}$.

the reduction reaction of dissolved oxygen has occurred irregularly due to the low applied potential at the early stage of polarization and the sea water disturbance by the cavity, and consequently violent fluctuations in current density were observed until the activation polarization was reached. Dissolved oxygen reduction reaction involves the suppression of increase in current density when reduction rate of oxygen on the electrode surface increases, and the oxygen ions in areas adjacent to metal surface are consumed fast. This is an electrochemical reaction dominated by diffusion, and its polarization is caused by a difference in concentration between the surface of the electrode and that of the electrolyte [9]. After that, there was a tendency to increase in current density as the potential had shifted into the active direction. Concentration polarization region by the dissolved oxygen reduction reaction existed in this experiment, but the severe fluctuations in current density are probably due to the supply of oxygen by cavities, resulting from forced circulation of sea water. At the low potential, a distinct difference in current density was not observed, but at potentials of less than $-2.0 \mathrm{~V}$, a higher current density was observed with increasing temperature.

Figure 4 compares the 3D microscope observation result and maximum damage depth following the anodic polarization and cathodic polarization experiments of 5083-O aluminum alloy at different sea water temperatures at the vibration amplitude of $10 \mu \mathrm{m}$.

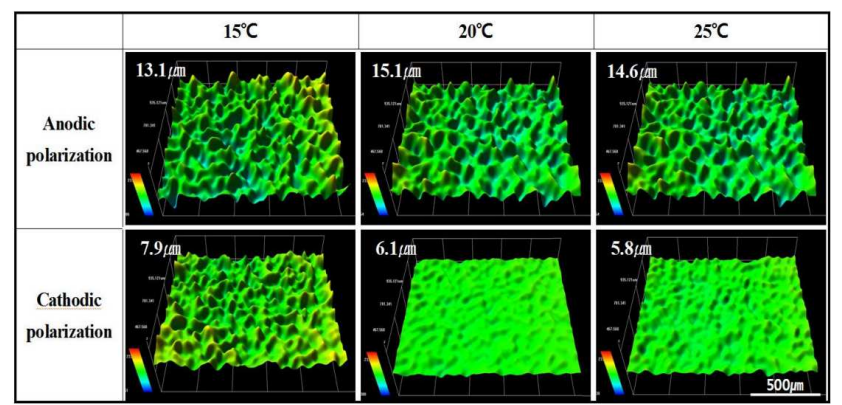

Fig. 4. Comparison of 3D analysis results after anodic and cathodic polarization of 5083-O $\mathrm{Al}$ alloy at different sea water temperatures, at vibration amplitude of $10 \mu \mathrm{m}$.

The 3D analysis has revealed the damage on the specimen surface caused by corrosion and elimination by continuous dissolution reaction under the condition of anodic polarization. In the case of aluminum, the low current density region is observed due to the formation of oxide film, caused by oxide reaction at the early stage of anodic polarization, which disturbs the charge transfer. However, a rough damaged surface was observed with continuous active solution reaction which had removed the oxide. The damage depth have shown the highest values at $20^{\circ} \mathrm{C}$, but were very similar at $25^{\circ} \mathrm{C}$. As a result, the variation of the damage rate with temperature after the anodic polarization experiment was not significant. In addition, the difference in current density on the polarization curves involves a difference of activation reaction of $\mathrm{Cl}^{-}$ion, rather than a difference in cavity impact pressure with temperature in a potentiodynamic experiment conducted in a short time. From the earlier studies, it is known that the dissolved oxygen in aqueous solutions becomes activated with increasing temperature, and simultaneously metal ion also becomes activated, consequently getting sensitized to corrosion [10]. This experiment has shown a very similar result since the electrochemical corrosion and cavitation damage worked in combination, and the temperature difference was not large. In the early stage of cathodic polarization, oxygen ion was consumed fast, as oxygen reduction rate on the surface had increased, and reduction reaction of dissolved oxygen occurred, indicating a steady current density. This region corresponds to a protection range as it shows low current density, but the damage is considered to have been induced by activation polarization in which hydrogen gas is generated by shifting to negative potential. In this research, hydrogen gas, which occurred by hydrogen over-potential during the activation polarization, may eliminate cavity impact pressure generated by ultrasonic vibratory device. Therefore, it is likely that only partial impact pressure is delivered to the surface, and induces slight damage. As a result, the difference in damage behavior is resulted from a difference in activation of $\mathrm{Cl}^{-}$ion with temperature. It seems that corrosion accelerates with the increasing temperature, and by partial delamination of the surface caused 
by the delivered impact pressure. The measurement of maximum damage depth revealed that the largest depth was obtained at $15^{\circ} \mathrm{C}$ and the lowest at $25^{\circ} \mathrm{C}$, however these values a very similar.

Figure 5 presents SEM images of the damaged surface and weight loss results, obtained after the anodic polarization and cathodic polarization experiments of 5083-O aluminum alloy at different temperatures in sea water, at the vibration amplitude of $10 \mu \mathrm{m}$.

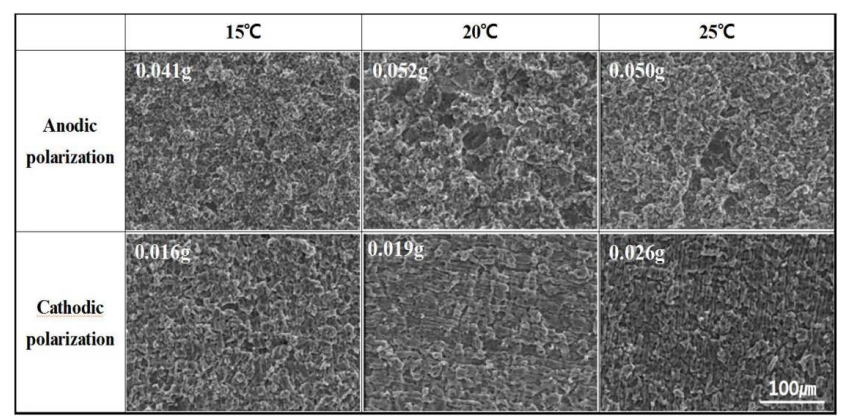

Fig. 5. Comparison of SEM morphologies and weight loss results after anodic and cathodic polarization for 5083-O Al alloy at different sea water temperatures at vibration amplitude of $10 \mu \mathrm{m}$.

Under the anodic polarization, a uniform damage has occurred at $15^{\circ} \mathrm{C}$ while pitting has occurred locally at $20^{\circ} \mathrm{C}$ and $25^{\circ} \mathrm{C}$. As a result, the surface damage was caused by corrosion damage due to active solution reaction and impact damage due to cavity, however it is estimated that there exists a difference in damage behavior, depending on the difference in $\mathrm{Cl}^{-}$ion activation degree with temperature. Generally, a material having face centered cubic (FCC) structure, such as aluminum, is not susceptible to deformation speed, compared to other materials [7]. Therefore, it undergoes the incubation period due to the formation of compressive residual stress caused by cavitation impact pressure in the early stage. After that, with continuous collision with cavity, pitting is created by plastic deformation and grows into a crater. Kling et al. investigated the damage induced by cavitation bubble collapse and found that a bubble divides in two small oval cavities and a microjet is produced somewhere in between them, moving towards the surface after starting to collapse from the surface of the specimen [11]. The cavitation damage, therefore, is considered to be a consequence of simultaneous action of impact pressure by cavity collapse and the microjet. In the cathodic polarization, most damage is caused by hydrogen evolution due to activation polarization. In this research, however, it was the damage from plastic deformation due to cavity impact pressure. During the activation polarization, hydrogen gas generated by the hydrogen overpotential collides with cavity, and thus cancels or repulses the impact. Therefore, impact pressure was not delivered to the surface of material directly, and thus partially delivered impact pressure is estimated to collide with the base metal, causing plastic deformation. Using such a technique, water cavitation peening, cavitation damage can be reduced. If excessive polarization is applied, however, the hydrogen permeation causes embrittlement of materials, which exacerbates the damage. Therefore, it is important to determine the limiting potential for corrosion protection. In contrast with the result of the weight loss, the damage depth became smaller with the elevating temperature. This is due to the relatively uniform damage distribution following the elimination of the microstructure, which had been delaminated by corrosion and activation reaction.

Figure 6 exhibits Tafel analysis results after polarization experiments for 5083-O aluminum alloy at different temperatures in sea water at the vibration amplitude of $10 \mu \mathrm{m}$.



Fig. 6. Polarization curves for Tafel analysis and results of 5083-O Al alloy for different sea water temperatures, at $10 \mu \mathrm{m}$ vibration amplitude.

The corrosion potential and corrosion current density at $15^{\circ} \mathrm{C}$ were $-1.087 \mathrm{~V}$ and $2.661 \times 10^{-5} \mathrm{~A} / \mathrm{cm}^{2}$, respectively, representing the lowest corrosion current density among the three studied conditions. On the other hand, both $20^{\circ} \mathrm{C}$ and $25^{\circ} \mathrm{C}$ experiments have shown a very similar tendency in which the corrosion potentials were $-1.133 \mathrm{~V}$ and $-1.156 \mathrm{~V}$, respectively, and the corrosion current densities were $3.225 \times 10^{-5} \mathrm{~A} / \mathrm{cm}^{2}$ and $4.152 \times 10^{-5} \mathrm{~A} / \mathrm{cm}^{2}$, respectively. Therefore, the corrosion current density tended to increase with the increasing temperature. The difference in the corrosion rate during electrochemical experiment in sea water was observed under the same vibration amplitude. It is considered to be due to the difference in $\mathrm{Cl}^{-}$ion activation and cavity impact pressure depending on temperature. As a result, corrosion damage is expected to be accelerated due to the synergistic effect of erosion and corrosion as the solution temperature is elevated. Tsyrulnyk drew the same conclusion from his research into the effect of temperature on corrosion, corrosion fatigue and cavitation damage using tap water [12]. 
Figure 7 shows the surface damage with cavitation time for 5083-O aluminum alloy in sea water at the vibration amplitude of $10 \mu \mathrm{m}$.



Fig. 7. SEM morphologies of 5083-O Al alloy after cavitation experiment during different time intervals at $10 \mu \mathrm{m}$ vibration amplitude.

In the case of 0.5 hour of cavitation, pit generation and combining of pits were observed simultaneously due to impact pressure caused by cavity. The number of pits has increased and then the pits were widely expanded during the one-hour-long cavitation. In general, the process of cavitation damage involves the plastic deformation accompanied by the work hardening in the early stage, showing the incubation period in which erosion becomes stagnant. It is considered to be $0.5 \mathrm{~h}$ in this experiment. The incubation period is the time before the fatigue effect, caused by the accumulation of cavitation impact energy, becomes noticeable. It is reported that this period heavily relies on fatigue strength among other mechanical properties of materials [13]. At two hours of cavitation, this tendency continued, and large damage to crater pit was created. At three hours of cavitation, the damage expanded into the area surrounding the crater pit. The pits after cavitation for five hours show mesh shapes, and micropits and cracks were observed inside large pits. It is suggested that this is because less bubbles are formed as they shift outwards from the center, and because small amounts of bubbles in a pit absorb the shock. At ten hours of cavitation, the middle-sized pits observed after five hours were merged, and broader and deeper damage was found on the entire specimen. As a result, after $0.5-1$ hour, the pits created by local damage were observed, however after two hours of cavitation the overall damage was produced and the pits have grown in size. In the previous research, a greater damage was induced by increasing the vibration amplitude and thus the cavity impact pressure [14]. In this study, it was observed that the cumulative impulse increased with the increasing exposure time of cavitation, resulting in greater damage. Cavitation damage in sea water typically involves erosion, and the synergistic effect of erosion and corrosion accelerates the damage. Metal ionizes to catalyze the dissolution reaction by anodic reaction in the pits, where cathodic hydrogen evolution is observed. Moreover, hydrogen gas bubbles produced by cathodic reaction are collapsed, producing microjets and ultrajets, resulting in mechanical damage. Consequently, the simultaneous action caused by collapse of cavitation bubbles and hydrogen gas in a corrosion environment accelerates the damage. Such synergistic effect is thought to be responsible for the physical collision.

Figure 8 presents dependencies of weight-loss and cavitation rate as functions of time in sea water for 5083-O aluminum alloy at the vibration amplitude of $10 \mu \mathrm{m}$.



Fig. 8. Weight-loss after cavitation experiment and cavitation rate of 5083-O Al alloy as functions of time at $10 \mu \mathrm{m}$ of vibration amplitude.

The weight-loss has shown a tendency of gradual increase with a steady rate throughout the experiment. On the other hand, cavitation rate has shown the highest value of $0.0274 \mathrm{~g} / \mathrm{h}$ by means of overall grain elimination at 0.5 hour. Then it sharply decreased after one hour to the value of $0.0056 \mathrm{~g} / \mathrm{hr}$. After one hour, because of cavitation peening effect accumulated earlier, a compressive residual stress has occurred and the hardness has increased. Thus, it is estimated that the cavitation rate was reduced owing to the improvement in cavitation resistance [15]. Afterwards, it has shown a tendency of a gradual decrease and by three hours, it showed the lowest value of $0.0029 \mathrm{~g} / \mathrm{h}$.

Figure 9 shows the 3D analysis of the surface as function of time for 5083-O aluminum alloy at the vibration amplitude of $10 \mu \mathrm{m}$.

The damaged surface shows that by 0.5 hour, the surface has become rough due to the damage caused by cavity. After one to two hours, this damage has accelerated and shows a more rough surface. As the damage combined, it became bigger and deeper. By three hours, more grown pits which are sharp and rough, can be observed. The maximum damage depth shows a steady growth, having $18.5 \mu \mathrm{m}$ after 0.5 hour, $24.5 \mu \mathrm{m}$ after one hour and $30.0 \mu \mathrm{m}$ after two hours. By the three hours, as pits grow in depth, they show a tendency of a smaller increment. To the end of the experiment, the damage 


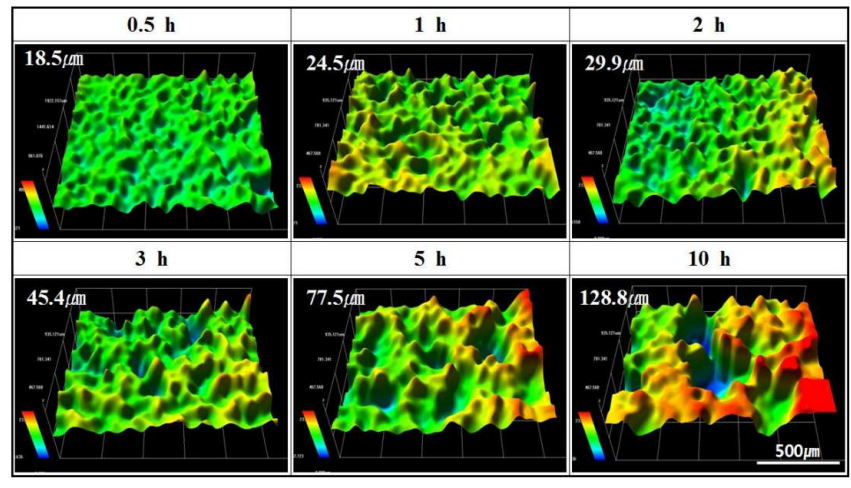

Fig. 9. 3D analysis of the surface and damage depth of 5083-O Al alloy after cavitation experiment for different time intervals at vibration amplitude of $10 \mu \mathrm{m}$.

depth has become larger at a fixed pit size and by ten hours, it showed damage depth of $128.8 \mu \mathrm{m}$. As a result, there is a tendency of a steady growth of the damage depth with time, and it is estimated that the gradation process, in which pits are combined, has an influence on growth rate of the maximum damage depth.

\section{Conclusion}

In this research, a hybrid test was conducted to assess the characteristics of 5083-O aluminum alloy in sea water at varied solution temperature and cavitation time. According to the cavitation-electrochemical experiment at various temperatures, the synergistic effects of corrosion by the $\mathrm{Cl}^{-}$ion in sea water and the impact pressure of cavitation were observed. Consequently, with the increasing temperature, the corrosion current density increases and may accelerate corrosion damage. According to the cavitation experiment at varied duration intervals, the cumulative impact energy delivered to the surface grows with the increasing exposure time, and thus the weight-loss has shown a steady increasing tendency. In conclusion the damage is proportionally greater with the increase in temperature and the exposure time. Exposure to high temperature is therefore recommended to be avoided, and optimal design is necessary to reduce the cavitation.

\section{Acknowledgments}

This research was a part of the project entitled "Construction of eco-friendly $\mathrm{Al}$ ship with painting, and maintenance/repairment free", funded by the Ministry of Oceans and Fisheries, Korea. This work was also supported by the New \& Renewable Energy of the Korea Institute of Energy Technology Evaluation and Planning (KETEP) grant funded by the Korea government Ministry of Trade, Industry \& Energy (No.20123010090020).

\section{References}

[1] J.A. Joyner, SAE Trans. 65, 337 (1956).

[2] S.J. Kim, S.J. Lee, Corros. Sci. Technol. 10, 101 (2011).

[3] H. Mochizuki, M. Yokota, S. Hattori, Wear 262, 522 (2007).

[4] H. Chen, J. Li, D. Chen, J. Wang, Wear 265, 692 (2008).

[5] J.H. Kim, K.S. Na, G.G. Kim, J.Y. Oh, C.S. Yoon, S.J. Kim, Mater. Sci. Eng. A 477, 204 (2008).

[6] E.A. Brujan, A.F.H. Al-Hussany, R.L. Williams, P.R. Williams, Wear 264, 1035 (2008).

[7] S.J. Kim, Metals and Materials Test Methods, DaeJin, Busan 2011 (in Korean).

[8] D.A. Jones, Principles and Prevention of Corrosion, Prentice-Hall, New Jersey 1996.

[9] H.R. Lee, Corrosion of Metals, Yeunkyung, Busan 2004 (in Korean).

[10] K.J. Kim, Corros. Sci. Technol. 17, 82 (1988).

[11] R.T. Knapp, J.W. Daily, F.G. Hammitt, Cavitation, McGraw-Hill, New York 1970.

[12] O.T. Tsyrul'nyk, Mater. Sci. 36, 136 (2000).

[13] Y.K. Zhou, F.G. Hammitt, Wear 86, 299 (1983).

[14] S.J. Lee, S.J. Kim, Corros. Sci. Technol. 11, 205 (2012).

[15] S.J. Lee, K.H Kim, S.J. Kim, Surf. Interface Anal. 44, 1389 (2012). 